\title{
Mortalidade por causas externas na região do ABCD paulista de 1996 a 2008: o problema dos "eventos de origem indeterminada" e a responsabilidade do médico legista
}

\section{Mortality due external injuries in $A B C D$ region of Sao Paulo from 1996 to 2008: the problem of undetermined origen events and the responsability of the coroner}

\author{
Raphael Aguiar Rodrigues da Costaํㅜ, Carmen Silvia Molleis Galego Miziara², \\ Ivan Dieb Miziara ${ }^{3}$
}

\begin{abstract}
Costa RAR, Miziara CSMG, Miziara ID. Mortalidade por causas externas na região do ABCD paulista de 1996 a 2008: o problema dos "eventos de origem indeterminada" e a responsabilidade do médico legista. Saúde, Ética \& Justiça. 2011;16(1):39-46.
\end{abstract}

\begin{abstract}
RESUMO: Introdução: A região do ABCD paulista apresenta índices elevados de morbimortalidade por causas externas. O estudo dessas variáveis é fundamental para a implantação de políticas públicas de prevenção e atendimento, sendo a causa básica da morte um importante elemento de estudo nas análises de mortalidade nos programas de saúde pública. O preenchimento equivocado da Declaração de Óbito acarreta notificação inadequada das mortes por estas causas, que passam a ser denominadas de "origem indeterminada". O médico legista desempenha papel fundamental neste processo. Objetivos: analisar a mortalidade por causas externas na região do ABCD paulista, de 1996 a 2008, e a incidência de lesões de "origem indeterminada". Metodologia: Estudo descritivo com base nos bancos de dados disponibilizados pelo Ministério da Saúde do Brasil referentes ao período entre1996 e 2008. Resultados: Ocorreram 26.966 mortes por causa externa na região do ABCD no período estudado. Entre as vítimas, predominaram jovens do sexo masculino. Os eventos de "origem indeterminada" sofreram elevação em São Bernardo do Campo (de 8,7\% para 19\%) e em Santo André (de 7,4\% para 21,6\%) entre 1996 e 2008. Discussão: Os dados acerca de mortalidade por causas externas na região do ABCD são similares aos do restante do país. Houve elevação de eventos de "causa indeterminada" ocorridos nos municípios de Santo André e de São Bernardo do Campo. Infelizmente, o excessivo número de óbito de causa indeterminada compromete a real identificação da causa da morte e, em consequência, interfere no delineamento do perfil epidemiológico da região. Conclusões: A identificação da causa dos óbitos, indiscutivelmente, pode auxiliar de forma determinante o desenvolvimento e implantação de políticas públicas adequadas.
\end{abstract}

DESCRITORES: Causas externas; Mortalidade; Responsabilidade pela informação; Causas de morte; Medicina Legal.

\footnotetext{
1. Acadêmico de Medicina na Faculdade de Medicina do ABC - Fundação do ABC.

2. Médica, Departamento de Medicina Legal, Ética Médica e Medicina Social e do Trabalho da Faculdade de Medicina da Universidade de São Paulo.

2. Professor Titular da Disciplina de Medicina Legal e Deontologia Médica da Faculdade de Medicina do ABC.

Endereço para correspondência: Rua Teodoro Sampaio, 352 Cj. 22. São Paulo-SP. CEP 05406-000.

E-mail:miz@uol.com.br
} 


\section{INTRODUÇÃO}

$\mathrm{N}$ os últimos anos, sobretudo a partir da década de oitenta, a taxa de morbimortalidade devido às causas externas tem assumido proporções significativas no mundo. Em 2003, representou 13\% de todos os óbitos ocorridos no Brasil' ${ }^{2}$. Em 2007, do total de 131.032 óbitos por causas externas no Brasil, $67 \%$ foram decorrentes de homicídios e de acidentes de trânsito Mortalidade é a variável característica das comunidades de seres vivos; refere-se ao conjunto dos indivíduos que morreram num dado intervalo do tempo. Representa o risco ou probabilidade que qualquer pessoa na população apresenta de poder vir a morrer ou de morrer em decorrência de uma determinada doença. É calculada pela taxas ou coeficientes de mortalidade, que é a medida do número de mortos em uma população em relação à população local e a um determinado período de tempo e representam o "peso" que os óbitos apresentam numa certa população 4 .

Segundo a OMS podemos classificar a mortalidade em três grandes grupos, sendo que o primeiro e o segundo incluem as doenças transmissíveis e não-transmissíveis, entre outros aspectos relativos às enfermidades, respectivamente ${ }^{5}$. O terceiro, todavia, aborda as causas de mortes não relativas a doença, tais como os acidentes de transporte, as agressões, as quedas e o suicídio 6 .

Por definição do Conselho Federal de Medicina, óbito por causa externa, ou não natural, é aquela que decorre da lesão provocada por violência (homicídio, suicídio, acidente, ou morte suspeita) qualquer que tenha sido o tempo entre o evento e a morte propriamente?

A partir de 1976, o Ministério da Saúde implantou o modelo único de DO, visando fornecer para o Sistema de Informações de Mortalidade (SIM) dados padronizados sobre a mortalidade. Estas informações passam a fazer parte da base de cálculo das estatísticas vitais e epidemiológicas do país. Com a padronização da DO houve um avanço no aspecto jurídico, pois o documento cumpre os ditames legais para as formalidades do sepultamento, conforme a Lei dos Registros Públicos no $60.15 / 73$. Conforme esclarece o Conselho Federal de Medicina (CFM), somente com o empenho e compromisso do médico com relação à veracidade, completitude e fidedignidade das informações registradas na DO estes objetivos serão atingidos, uma vez que é o profissional responsável pelas informações. ${ }^{7}$
O Conselho ainda enfatiza que a DO ultrapassa a importância legal, pois é instrumento utilizado para conhecer a situação de saúde da população e gerar ações visando sua melhoria com base na DO emitida pelo médico. A importância da Declaração de Óbito (DO) é igualada apenas à Certidão de Nascimento. Na tentativa de obter dados confiáveis a respeito da real causa do óbito por causa externa, a OMS orienta que não basta conhecer a natureza da lesão, mas é fundamental esclarecer qual foi o agravo que culminou com a morte. Exemplificando, se a lesão decorreu de politraumatismo por atropelamento causado por motocicleta, a natureza da lesão foi o politraumatismo e o fator que precisa ser abordado preventivamente é o atropelamento ${ }^{8}$. Complementa ainda que, os óbitos precisam ser codificados de acordo com a causa básica conforme o Capítulo XX da Classificação Estatística Internacional de Doenças e Problemas Relacionados à Saúde, Décima Revisão ${ }^{9}$.

Segundo o Manual de Instruções para Preenchimento da Declaração de Óbito, publicado pela Fundação Nacional de Saúde, FUNASA, 2001, causa básica é a "doença ou lesão que iniciou a cadeia de acontecimentos patológicos que conduziram diretamente à morte, ou as circunstâncias do acidente ou violência que produziram a lesão fatal ${ }^{10}$.

Outro fato a ser considerado é o impacto da morbimortalidade por causas externas na Saúde Pública brasileira, uma vez que as internações por estas causas, mesmo sendo por um período menos prolongado do que a média das outras causas (tempo médio de internação de 5,2 dias, comparado com 6,1 dias para todas as causas), tendem a consumir mais recursos. Como consequência, apresentam um gasto/dia $60 \%$ superior à média geral das hospitalizações pagas pelo Sistema Único de Saúde (SUS). Com relação à mortalidade, o impacto econômico foi analisado por meio do indicador Anos Potenciais de Vida Perdidos. Os acidentes e violências representaram cerca de 2,6 milhões de anos de vida perdidos, em 1981, e 3,4 milhões, em 199111,12,13,14.

A região do $A B C D$ (São Bernardo do Campo, Diadema, Santo André, Mauá, São Caetano do Sul, Ribeirão Pires e Rio Grande da Serra), devido à alta concentração populacional, há vários anos apresenta índices elevados de morbimortalidade por causas externas ${ }^{1,3,4}$. Assim, os objetivos deste estudo são: analisar os dados referentes à mortalidade por causas externas na região do ABCD paulista no período de 1996 a 
2008 e analisar a incidência de lesões de "origem indeterminada" como componente da taxa de mortalidade por causas externas.

\section{METODOLOGIA}

Este estudo descritivo foi realizado com base em pesquisa nos bancos de dados disponibilizados pelo Ministério da Saúde do Brasil, DATASUS (http:// www2.datasus.gov.br/DATASUS). Os dados foram coletados individualmente para cada município da região do $A B C D$, entre1996 e 2008, sendo de especial importância ao estudo os dados relativos à incidência de lesões de "origem indeterminada".

\section{RESULTADOS}

Foram registrados nos bancos de dados oficiais, entre 1996 e 2008, 26.966 mortes de causa externa nos sete municípios da região do $A B C D$ paulista (Tabela 1), sendo que a microrregião representada pelos municípios de Santo André, São Bernardo do Campo, São Caetano do Sul e Diadema teve um ponto máximo de elevação no número de óbitos totais por causa externa no período entre 1996 e 1999, sofrendo, a seguir, queda considerável (de 40,88\%), progressiva, a partir do ano de 2000 até o ano de 2008 (Tabela 1 e Gráfico 1).

Tabela 1. Distribuição do número de óbitos por causa externa de acordo com o município

\begin{tabular}{lllllllll}
\hline Ano & SBC & DD & Total de óbitos por causa externa por cidade & SA & Mauá & SCS & RP & RGS \\
\hline $\mathbf{1 9 9 6}$ & 715 & 652 & 611 & 344 & 95 & 97 & 32 & 2.541 \\
$\mathbf{1 9 9 7}$ & 684 & 683 & 585 & 320 & 97 & 71 & 34 & 2.474 \\
$\mathbf{1 9 9 8}$ & 700 & 665 & 542 & 335 & 92 & 66 & 43 & 2.423 \\
$\mathbf{1 9 9 9}$ & 741 & 682 & 593 & 403 & 79 & 95 & 50 & 2.643 \\
$\mathbf{2 0 0 0}$ & 710 & 554 & 614 & 388 & 85 & 79 & 35 & 2.465 \\
$\mathbf{2 0 0 1}$ & 572 & 496 & 550 & 378 & 70 & 73 & 30 & 2.182 \\
$\mathbf{2 0 0 2}$ & 532 & 536 & 519 & 379 & 89 & 71 & 32 & 2.158 \\
$\mathbf{2 0 0 3}$ & 563 & 466 & 509 & 310 & 83 & 74 & 16 & 2.021 \\
$\mathbf{2 0 0 4}$ & 540 & 401 & 496 & 314 & 85 & 64 & 31 & 1.931 \\
$\mathbf{2 0 0 5}$ & 459 & 417 & 407 & 281 & 67 & 61 & 33 & 1.725 \\
$\mathbf{2 0 0 6}$ & 418 & 283 & 417 & 227 & 65 & 65 & 14 & 1.489 \\
$\mathbf{2 0 0 7}$ & 396 & 271 & 352 & 239 & 57 & 74 & 23 & 1.412 \\
$\mathbf{2 0 0 8}$ & 389 & 316 & 394 & 252 & 70 & 59 & 22 \\
\hline
\end{tabular}

SBC: São Bernardo do Campo; DD: Diadema; SA: Santo André; SCS: São Caetano do Sul; RP: Ribeirão Pires; RGS: Rio Grande da Serra. Fonte: MS/SVS/DASIS - Sistema de Informações sobre Mortalidade - SIM

Gráfico 1. Taxas de mortalidade por causas externas na microrregião de saúde do Grande ABC em números de óbitos totais por residência

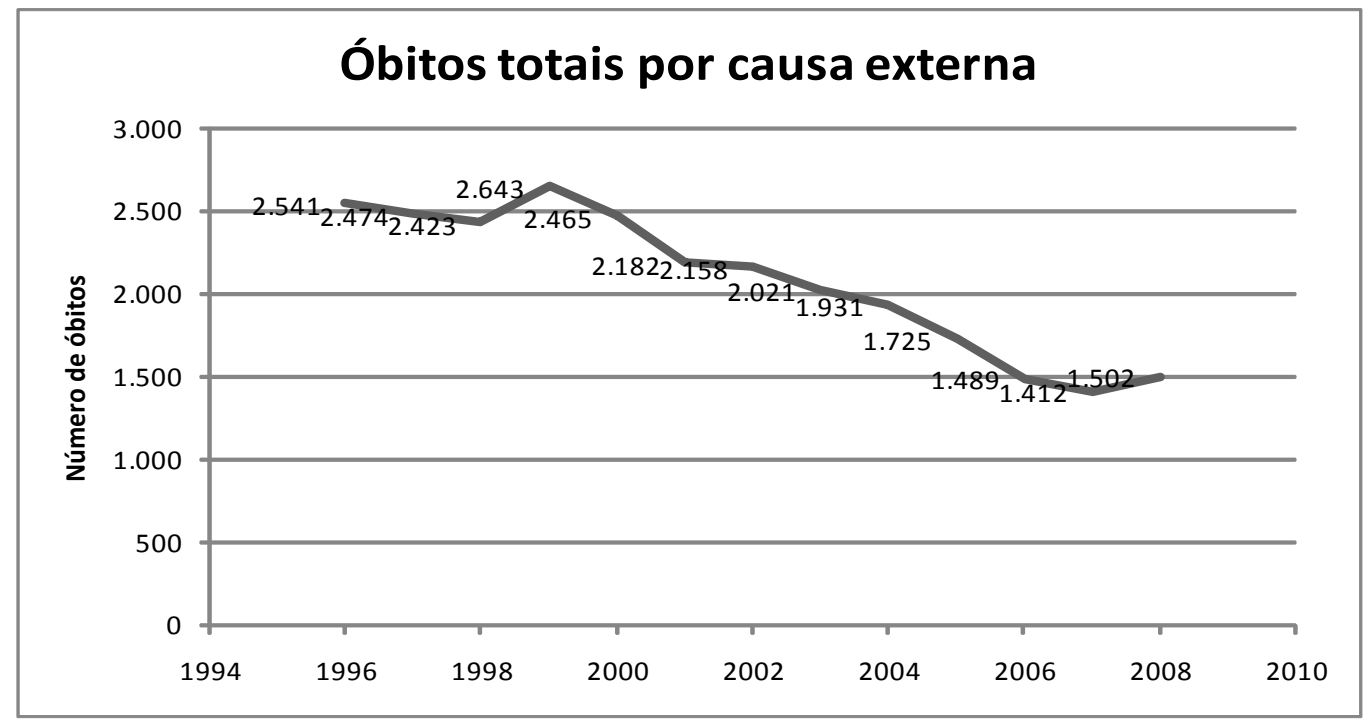

Fonte: MS/SVS/DASIS - Sistema de Informações sobre Mortalidade - SIM

Por outro lado, foi notado, de modo mais específico, que os dados relativos aos municípios da microrregião demonstram declínio nos valores absolutos de óbitos por causas externas de 1996 até 2006, quando passam a apresentar elevação (Gráfico 2). 
Costa RAR, et al. Mortalidade por causas externas na região do ABCD paulista de 1996 a 2008.

Gráfico 2. Taxa de mortalidade por causas externas na microrregião de saúde da Grande $A B C$ em números de óbitos totais por residência

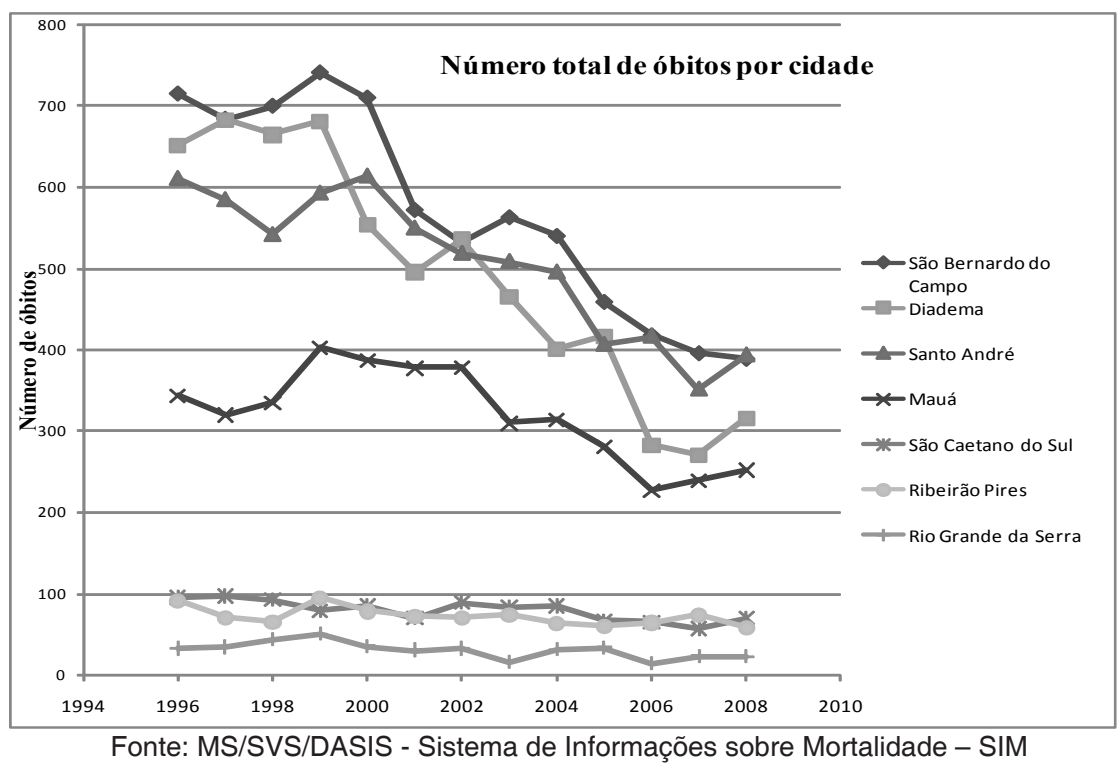

Nos índices registrados em 1996 e 2008 foi observada a queda no número de mortes por causas externas em todos os municípios, sendo que Diadema teve a maior redução $(51,53 \%)$, seguido por São Bernardo do Campo $(45,49 \%)$ e por Ribeirão Pires (35,87\%) (Tabela 2). Mesmo com a maior redução percentual no número de óbitos, Diadema permanece entre os três municípios com maior incidência de óbitos por causas externas dentre aqueles pesquisados.

Tabela 2. Óbitos por causas externas por municípios

Óbitos por Causas Externas - Municípios do ABC (1996 e 2008) - Valores Absolutos e Relativos

\begin{tabular}{lccc} 
Município & $\mathbf{1 9 9 6}$ & $\mathbf{2 0 0 8}$ & Redução percentual \\
\cline { 2 - 3 } Diadema & 652 & 316 & $-51,53 \%$ \\
Mauá & 344 & 252 & $-26,74 \%$ \\
Ribeirão Pires & 92 & 59 & $-35,87 \%$ \\
Rio Grande da Serra & 32 & 22 & $-31,25 \%$ \\
Santo André & 611 & 394 & $-35,52 \%$ \\
São Bernardo do Campo & 715 & 389 & $-45,59 \%$ \\
São Caetano do Sul & 95 & 70 & $-26,32 \%$ \\
Total & 2.541 & 1.502 & $-40,89 \%$ \\
\hline
\end{tabular}

Fonte: MS/SVS/DASIS - Sistema de Informações sobre Mortalidade - SIM

A análise das causas de morte realizada de acordo com os grupos da Classificação Internacional das Doenças - $10^{\underline{a}}$ revisão (CID10) está exposta na Tabela 3. Sob a luz desses dados, observa-se que a maior causa, nos três municípios, tanto no ano de 1996 quanto no ano de 2008 são as agressões (X85-Y09), seguidas de acidentes de transportes (V01-V99). No entanto, verifica-se também que as agressões possuem, em todos os casos, maior percentual de redução de número de óbitos. O número de notificações sem a que a determinação da causa externa da morte fosse determinada mostrou padrão ascendente nos municípios de São Bernardo do Campo e de Santo André. Na primeira cidade, a porcentagem passou de $8,7 \%$ para $19 \%$, mais que o dobro, nos anos de 
Costa RAR, et al. Mortalidade por causas externas na região do ABCD paulista de 1996 a 2008.

1996 e 2008, respectivamente. Em Santo André, estes valores foram mais expressivos, passando de $7,4 \%$ para $21,6 \%$, portanto, aproximadamente o triplo.

Tabela 3. Óbitos por causas externas por municípios e segundo a CID-10

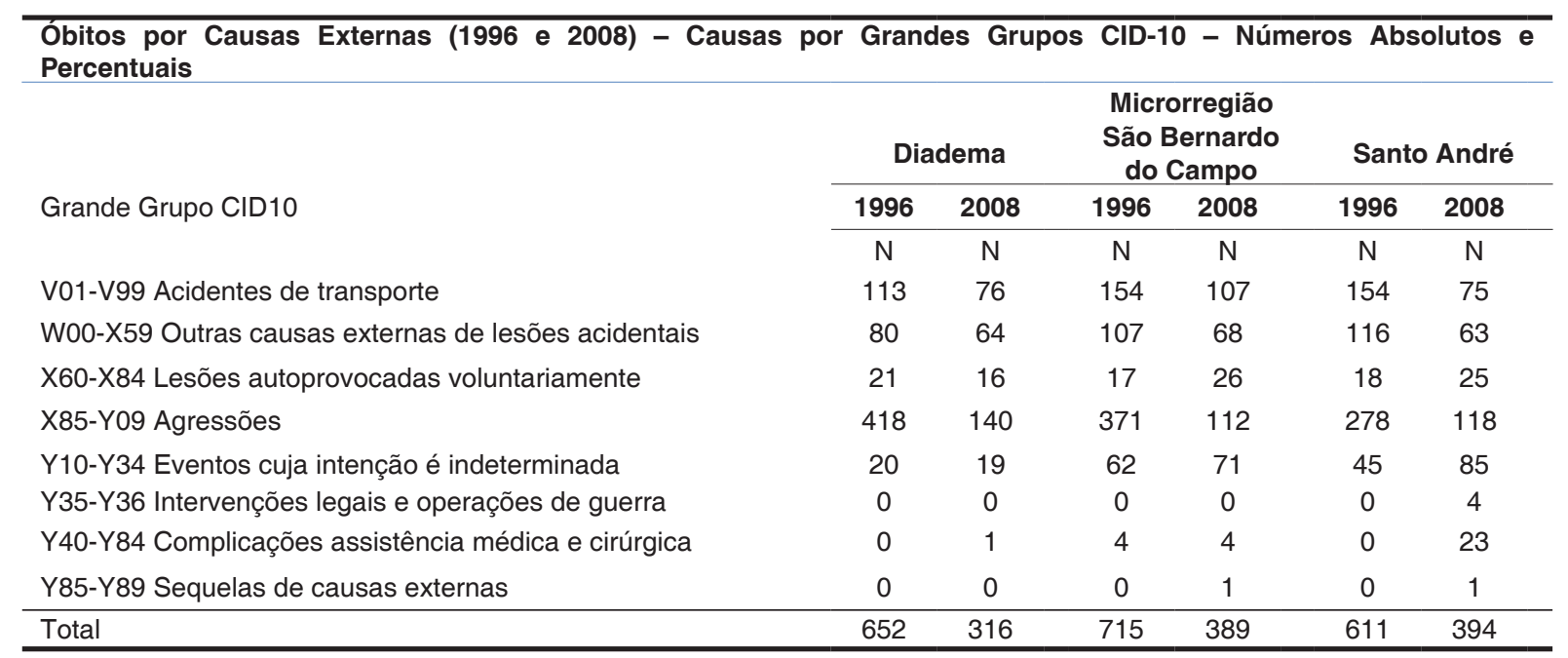

Fonte: MS/SVS/DASIS - Sistema de Informações sobre Mortalidade - SIM

A Tabela 4 expõe a distribuição de mortes de causas externas de acordo com a idade da vítima à época da ocorrência. A análise destes dados mostra o predomínio de jovens entre 15 e 39 anos (Tabela 4 e Gráfico 3), mas evidencia valores em declínio ao se comparar os anos de 1996 e
2008. A faixa etária correspondente ao intervalo entre 15 e 39 anos representou $69 \%$ de todas as vitimas, em 1996, enquanto que, em 2008, essa proporção foi de $56,7 \%$. Também foi verificada a grande predominância do sexo masculino (Tabela 5).

Gráfico 3. Taxas de Mortalidade por Causas Externas na Microrregião de Saúde do Grande ABC em Números de Óbitos Totais por Residência, de acordo com a faixa etária

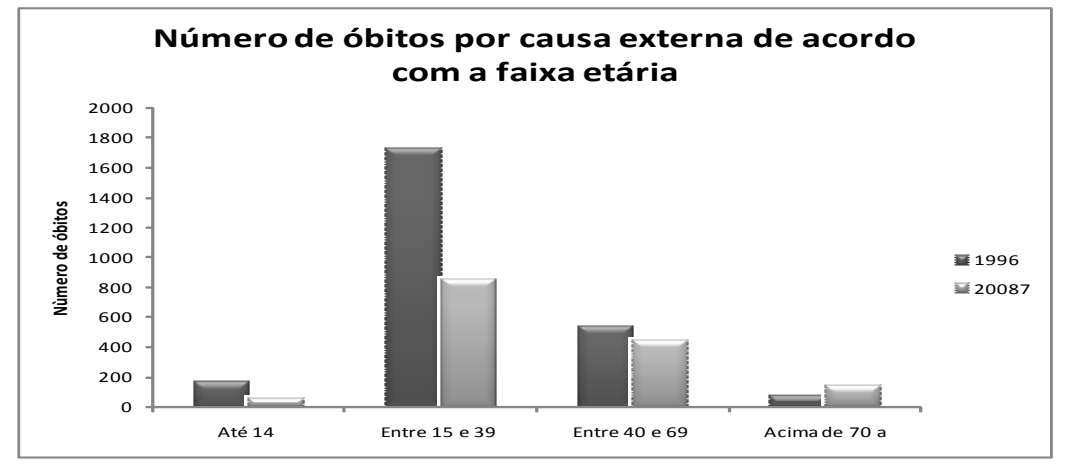

Fonte: MS/SVS/DASIS - Sistema de Informações sobre Mortalidade - SIM

Tabela 4. Óbitos por faixa etária devido a causas externas

Óbitos por Causas Externas por Fase da Vida - Municípios do ABCD (1996 e 2008) - Números Absolutos e queda percentual

\begin{tabular}{|c|c|c|c|}
\hline Faixa etária (anos) & 1996 & 2008 & $\%$ \\
\hline Até 14 & 164 & 60 & $-63,41 \%$ \\
\hline Entre 15 e 39 & 1725 & 849 & $-50,78 \%$ \\
\hline Entre 40 e 69 & 537 & 445 & $-17,13 \%$ \\
\hline Acima de 70 a & 75 & 145 & $+95,95 \%$ \\
\hline
\end{tabular}

Fonte: MS/SVS/DASIS - Sistema de Informações sobre Mortalidade - SIM 
Costa RAR, et al. Mortalidade por causas externas na região do ABCD paulista de 1996 a 2008.

Tabela 5. Distribuição do número de óbitos por causas externas de acordo com o sexo

\begin{tabular}{lccc}
\hline \multicolumn{3}{l}{$\begin{array}{l}\text { Óbitos por causas externas - Municípios do ABCD (1996 e 2008) - Por Sexo - Números absolutos e } \\
\text { percentuais do declínio }\end{array}$} & \multicolumn{2}{l}{ (1996 } & 2008 & $\%$ \\
\hline Fator & 2243 & 1229 & $-45,20 \%$ \\
Sexo Masculino & 298 & 273 & $-8,38 \%$ \\
Sexo Feminino & 2541 & 1502 & $-40,88 \%$ \\
Total & $88,27 \%$ & $81,82 \%$ & $-7,77 \%$ \\
\hline
\end{tabular}

Fonte: MS/SVS/DASIS - Sistema de Informações sobre Mortalidade - SIM

\section{DISCUSSÃo}

Segundo Minayo ${ }^{15}$, a categoria causas externas é operativa, sendo de valor para as organizações internacionais de saúde e sociais identificarem os principais aspectos relacionados a estas mortes e desta maneira fornecer sugestões aos governos nacionais e locais a respeito do fenômeno social da violência que provoca a morte, podendo então ser alvo de intervenções e comparações. ${ }^{15}$ Portanto, a análise epidemiológica deste fenômeno depende da fidedignidade dos dados fornecidos aos bancos de dados oficiais. A autora complementa que, na década de 90 mais de um milhão de pessoas morreram por causas externas, sendo que os homicídios lideraram com 400 mil casos, seguidos pelos acidentes de trânsito e suicídio.

Nos anos 2000 foi notado declínio destes números, principalmente nos últimos cinco anos. Neste estudo fica confirmada a maior probabilidade de mortes por causas externas em jovens do sexo masculino (80\%), dados estes coincidentes com os do Instituto Brasileiro de Geografia e Estatística (IBGE), pois em 2004 a mortalidade dos jovens do sexo masculino entre 20 e 24 anos era quatro vezes maior que o do sexo feminino, em São Paulo, nesta mesma época as chances eram seis vezes maiores, em 1980 esta proporção era inferior, corespondendo apenas de 2 para $1^{16}$.

As causas externas de óbitos (conforme determinadas pela CID 10) representam a terceira causa de óbitos no Brasil ${ }^{4}$. Em São Paulo foram registrados, em 2002, 32.328 mortes por causas externas, sendo o coeficiente de mortalidade de $84,6 / 100.000$ habitantes, variando de acordo com o sexo $(148,2 / 100.000$ entre os homens e $23,6 / 100.000$ entre as mulheres), portanto o risco relativo para indivíduos do sexo masculino foi 6,3 vezes maior que para os do sexo feminino, fato também observado no presente estudo ${ }^{6}$. Outro dado concordante com nosso estudo foi a maior taxa nas faixas etárias mais baixas. No Estado de São Paulo, a maior incidência $(43 \%$ de todas as vítimas) foi entre 15 e 29 anos e, em nosso grupo de estudo, foi de 15 até 39 anos, correspondendo a valores de $69 \%$ em 1996 e 56,7\% em 2008. Este resultado já era esperado, devido ao fato da maior probabilidade desses indivíduos sofrerem agressão e acidentes de transportes, em comparação com as demais faixas etárias, e acompanha uma tendência relevante no resto do país.

Entretanto, há que se notar o aumento significativo dessas causas de óbito na população acima dos 70 anos. A explicação pode ser dada pelo fato da população dessa faixa etária ter aumentado consideravelmente nesse período, $49,3 \%$ segundo CENSO $2000-2010^{10}$, aumentando assim o número de pessoas idosas suscetível a óbitos por causas externas ${ }^{16}$.

Em relação ao sexo, notamos predomínio elevado da população masculina nessa casuística. Pode-se argumentar que existe maior exposição do indivíduo de sexo masculino aos fatores de risco, embora exista a possibilidade de ocorrer aumento do número de mortes de mulheres devido ao maior ingresso destas no mercado de trabalho, o que as torna mais suscetíveis aos riscos de óbitos por causas externas ${ }^{11}$.

De todos os dados apresentados, dois deles chamam bastante à atenção. O primeiro é a tendência ascendente da curva de eventos por causas externas a partir de 2008 nos municípios de Santo André, Diadema e Mauá, conforme se pode visualizar no Gráfico 2. Esta tendência pode simplesmente estar acompanhando as mudanças 
nos números populacionais, mas, o que é mais preocupante, pode significar (após um período de queda nos números), que as políticas públicas de prevenção já não estejam sendo aplicadas com tanta ênfase e rigor nestas cidades.

Além disso, outro dado é extremamente relevante: a elevação de eventos de "causa indeterminada" ocorridas nos municípios de Santo André e São Bernardo do Campo no período estudado. Para efeito de comparação, os "eventos de causa indeterminada" (no mesmo intervalo de tempo) representaram $12 \%$ dos óbitos por causas externas no Estado de São Paulo índice muito abaixo daqueles apresentados pelos municípios citados. Obviamente, a consequência mais importante e indesejada, do ponto de vista epidemiológico, deste tipo de ocorrência é a subnotificação da real incidência de óbitos devido às causas externas na região pesquisada.

Vale ressaltar que é indiscutível a importância do médico legista quanto ao fornecimento de informações úteis na programação e implantação de medidas públicas saneadoras. É responsabilidade ética do médico o assinalamento de todas as informações contidas na DO, não devendo assinar DO em branco ou deixar declarações previamente assinadas; devendo ele, inclusive, verificar se todos os itens de identificação estão devida e corretamente preenchidos. Acrescente-se ainda que também é competência do médico seguir os preceitos legais e epidemiológicos vigentes, permitindo desta forma a identificação do processo mórbido e possibilitando a ação da vigilância quanto a medidas saneadoras. Todas as recomendações acima descritas fazem parte do Manual de Instrução para o Preenchimento da Declaração de Óbito, elaborado pelo Ministério da Saúde - Fundação Nacional da Saúde (FUNASA), em $2001^{10}$.

O manual de preenchimento de declaração de óbito do Ministério da Saúde, no item 1 do Bloco VIII -Causas externas-, também orienta e esclarece o médico sobre a boa prática de preenchimento indicando que as informações são de caráter estritamente epidemiológico. Ao se manifestar quanto a estas "circunstâncias", o médico legista deve assinalar (campo 56) se estas foram devidas a acidente, suicídio, homicídio, outra ou ignorada. No campo 57 deve assinalar se este acidente foi devido ou não ao trabalho, ou se é ignorada a relação. Por fim, no campo 58, correspondente à fonte da informação, o médico perito informa por qual meio foi obtida a informação: se boletim de ocorrência, hospital, família, outra ou ignorada. Deve-se ressaltar que na própria declaração encontra-se o lembrete de que essas "informações são de caráter estritamente epidemiológico", devendo o perito se orientar pela classificação da CID 10 - capítulo XX.

O Ministério da Saúde e Conselho Federal de Medicina estabelecem que é dever dos médicos: 1) preencher os dados de identificação com base em um documento da pessoa falecida; na ausência de documento, caberá, á autoridade policial, proceder o reconhecimento do cadáver; 2) registrar os dados na DO, sempre, com letra legível e sem abreviações ou rasuras; 3) registrar as causas da morte, obedecendo ao dispositivo nas regras internacionais, anotando um diagnóstico por linha e o tempo aproximado entre o inicio da doença e a morte; e 4) revisar se todos os campos estão preenchidos corretamente, antes de assinar ${ }^{7}$.

\section{CONSIDERAÇÕES FINAIS}

As elevadas taxas de mortes de causas externas nas cidades de São Bernardo do Campo, Diadema e Santo André, acima da média do Estado de São Paulo e por apresentarem, a partir de 2008, curva ascendente de incidência deste tipo de evento, sugerem a necessidade de medidas públicas preventivas no tocante à redução da mortalidade. Mesmo tendo bons percentuais de redução no período, ainda figuram entre as cidades com maiores números de óbitos devidos a causas não naturais. O estudo também confirmou as informações presentes na literatura de que o grupo com maior número de óbitos envolve o indivíduo jovem, predominantemente do sexo masculino.

Assim, segundo os dados coletados, concluímos que a microrregião do Grande ABCD vivencia a mesma problemática de muitas outras regiões do Brasil, consequente às políticas públicas, possivelmente inadequadas, no enfrentamento de mortes por causas externas, que poderiam ser evitadas em proporções significativas.

Além disso, o alto percentual de "eventos de origem indeterminada" detectado neste estudo sugere que o preenchimento do campo 58 do bloco VIII da DO, com dados específicos para cada caso, poderia fornecer informações úteis ao sistema de vigilância epidemiológica, tornando-se um instrumento de relevante importância social que talvez contribuísse para reduzir, por meio de políticas públicas de prevenção, a incidência de mortes por causas externas no Brasil. 
Costa RAR, et al. Mortalidade por causas externas na região do ABCD paulista de 1996 a 2008.

Costa da RAR, Miziara CSMG, Miziara ID. Mortality due to external injuries in ABCD region of Sao Paulo from 1996 to 2008: the problem of undetermined origens events and the responsability of the coroner. Saúde, Ética \& Justiça. 2011;16(1):39-46.

ABSTRACT: Introduction: The region of ABCD has high rates of morbidity and mortality due to external causes. The study of these variables is critical to the implementation of public policies for prevention and care. Fail in filling death certificate entails inadequate reporting of deaths due to these causes, which are now referred to as "undetermined". The medical examiner plays a key role in this process. Objectives: To examine mortality due to external causes in the ABCD region of Sao Paulo, from 1996 to 2008, and the incidence of injuries to "undetermined" cause. Methodology: A descriptive study based on databases provided by the Ministry of Health of Brazil between 1996 and 2008. Results: There were 26,966 deaths due to external causes in the ABCD region in the period studied. The victims included mostly young males. The events of "undetermined origin" had increased in Sao Bernardo do Campo (8.7\% to 19\%) and Santo Andre (from 7.4\% to 21.6\%) between 1996 and 2008. Discussion: The data on mortality due to external causes in the ABCD region are similar to those of the rest of the country. The elevation of the events of "undetermined cause" occurred in the municipalities of Santo Andre and Sao Bernardo do Campo. Unfortunately, the excessive number of deaths of undetermined cause may compromise the real identification of the cause of death and, consequently, interferes with the design of the epidemiological profile of the region. Conclusions: Identifying the cause of death can help in a determinant way the development and implementation of appropriate public policies, aimed at reducing deaths classified as external causes.

KEY WORDS: External causes; Mortality; Duty to warn, Forensic medicine.

\section{REFERÊNCIAS}

1. Laurenti R, Mello Jorge MHP, Gotlieb SLD Informação em mortalidade: o uso das regras internacionais para a seleção da causa básica. Rev Bras Epidemiol. 2009;12:195-203.

2. Secretaria de Vigilância em Saúde. Departamento de Análise de Situação de Saúde. Vigilância em saúde: dados e indicadores selecionados - ano $3, n^{\circ} 3$, nov. 2005. Brasília: Ministério da Saúde; 2003.

3. Brasil. Ministério da Saúde - DATASUS. Disponível em: http://tabnet.datasus.gov.br/tabdata/sim/dados/ indice.htm.

4. Centro de Vigilância Sanitária. Disponível em: http:// www.cvs.saude.sp.gov.br/pdf/epid_visa

5. World Health Organization. Available from: http://www. who.int/countries/bra/es

6. Nogueira RP. Mortalidade por três grandes grupos de causa no Brasil IPEA. Políticas Sociais Acompanhamento e Análise. 2004; 9:139-45.

7. Brasil. Ministério da Saúde e Conselho Federal de Medicina. A declaração de óbito: documento importante e necessário. Série A. Normas e manuais técnicos. Brasília: Distrito Federal; 2006.

8. Mello Jorge MHP; Gotlieb SLD; Laurenti R. O sistema de informações sobre mortalidade: problemas e propostas para o seu enfrentamento II - Mortes por causas externas. Rev Bras Epidemiol. 2002;5(2):21223.

Recebido em: 06/06/2011

Aprovado em: 30/06/2011
9. Organização Mundial da Saúde. CID-10. Classificação estatística internacional de doenças e problemas relacionados à saúde. $10^{a}$ rev. São Paulo: EDUSP; 1996.

10. Fundação Nacional de Saúde. Manual de instruções para o preenchimento da Declaração de Óbito. 3a ed. Brasília: Funasa; 2001. p.44.

11.Brasil. Ministério da Saúde. Sistema de Informação sobre Mortalidade [citado em 20 jun. 2011]. Disponível em: http://www2.datasus.gov.br/DATASUS. IBGE. Indicadores Sócio-demográficos e saúde no Brasil. 2009 [citado em 20 jun. 2011]. Disponível em: http:// www.ibge.gov.br.

12. lunes RF. III - Impacto econômico das causas externas no Brasil: um esforço de mensuração. Rev Saúde Pública. 1997;31(Supl. 4):38-46.

13. Brasil. Ministério da Saúde. Sistema de Informações sobre Mortalidade. DATASUS. Disponível em: http:// portal.saude.gov.br/portal/saude http://tabnet. datasus.gov.br/tabdata/sim/dados/indice.htm).

14.Portal da Saúde. SUS. Disponível em: http://portal. saude.gov.br/portal/saude.

15. Minayo MCS. Seis características das mortes violentas no Brasil. Rev Bras Est Pop. 2009;26(1):135-40.

16. Instituto Brasileiro de Geografia e Estatística - IBGE. Disponível em: www.ibge.gov.br/home/presidencia/ noticias/noticia. 